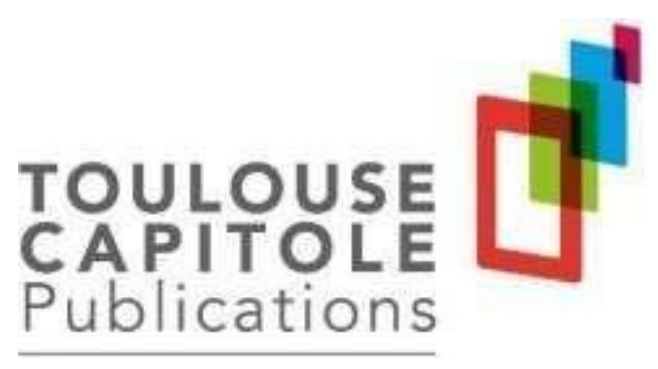

« Toulouse Capitole Publications » est l'archive institutionnelle de l'Université Toulouse 1 Capitole.

Le cinquantième anniversaire de la Constitution de l'île Maurice : le syncrétisme constitutionnel d'une jeune République

\title{
BLANC DIDIER
}

Référence de publication : BLANC (D.), « Le cinquantième anniversaire de la Constitution de l'île Maurice : le syncrétisme constitutionnel d'une jeune République », Revue française de droit constitutionnel, $\mathrm{n}^{\circ}$ 117, 2019.

Pour toute question sur Toulouse Capitole Publications, contacter portail- publi@ut-capitole.fr 


\section{Le cinquantième anniversaire de la Constitution de l'île Maurice : le syncrétisme constitutionnel d'une jeune République}

«Le 12 mars 1968, un nouveau nom que peu connaissaient jusque-là est apparu sur la liste des États indépendants : l'île Maurice, ancienne colonie britannique ${ }^{1}$. »

La Constitution du 12 mars 1958 à la suite de la révision constitutionnelle en vigueur au 12 mars $1992^{2}$, au jour de son vingt-quatrième anniversaire, s'ouvre sur un article $1^{\text {er }}$ posant que : « Maurice est un État souverain et démocratique dénommé République de Maurice. » L'affirmation principielle de la souveraineté, caractère distinctif de l'État ne surprend guère, pas plus que ses aspirations démocratiques tant elles forgent la règle commune dans un monde où les vastes empires coloniaux occidentaux laissent place aux indépendances nationales, manifestation tangible d'une liberté des peuples à disposer librement d'eux-mêmes. De la possession de l'île par la Hollande, suivie par la France puis enfin par la Couronne britannique, Maurice a connu un long processus se refermant dans sa dernière période par le passage en 1948 de l'autonomie interne à l'indépendance en $1968^{3}$. Ces vingt années charnières dans l'histoire mauricienne furent marquées par plusieurs étapes conduisant à l'indépendance après que son principe fut voté le 21 août 1967 par l'assemblée élue à la suite d'élections générales tenues le 7 août $1967^{4}$. La Constitution promulguée le 12 mars 1968 par le Mauritius Independence Order ${ }^{5}$, est muette quant à la forme du nouveau régime. Ce silence a pu être vu comme la manifestation d'une résurgence coloniale, contaminant au-delà l'ensemble du nouveau texte ${ }^{6}$. Quoi qu'il en soit, la République n'est pas contemporaine de l'indépendance, la Constitution mauricienne est un legs du régime britannique, « octroyée par l'ancienne puissance coloniale ${ }^{7} »$, si bien qu'il est permis de parler de seconde naissance à propos de l'instauration de la République. À l'instar de plusieurs territoires ayant quitté le giron de la Couronne Britannique, l'île Maurice a finalement choisi la forme républicaine du gouvernement comme vecteur de son indépendance, c'est-à-dire a minima un régime politique et constitutionnel dans lequel la dévolution du pouvoir ne relève pas de l'hérédité et ne saurait être « viagère ${ }^{8}$ ».

Il ne s'agit pas ici de retracer le passage d'un état à l'État ${ }^{9}$ mais de souligner la puissance du modèle républicain dans le constitutionnalisme contemporain. Depuis plus de deux siècles, la République qu'elle soit rêvée ou mythifiée importe peu - quels qu'en soient les contours et atours, opère une attraction à la fois à la faveur de changements poli- tiques et juridiques internes à l'État et, comme c'est le cas s'agissant de Maurice, dans un processus de décolonisation sur le temps long. De là à considérer que la République est la forme parfaite dans un sens premier et achevée du gouvernement des Hommes... En tout état de cause, est donné corps au point de vue des constitutions « républicaines », se considérant comme le stade suprême et ultime de l'évolution des régimes politiques.

Ce mouvement est ancien et pour l'essentiel univoque, il puise ses racines dans l'Antiquité, Athènes et Rome chassent des royautés archaïques et jettent les bases respectivement de la Démocratie et de la République qu'on se gardera toutefois de rapprocher des notions que recouvrent ces termes dans le droit constitutionnel contemporain ${ }^{10}$. Ses manifestations les plus tangibles et prospères ont aussi bien guidé les pères fondateurs des États-Unis que les acteurs de la révolution française qui durent enjamber les siècles pour nommer la forme politique qu'ils projetaient de bâtir. Hier, aujourd'hui et pour longtemps, une constitution ne se revendiquant pas républicaine, au prix parfois de l'artifice pour masquer ses lacunes démocratiques ${ }^{11}$, fait figure d'anomalie. 
La Constitution mauricienne s'inscrit tardivement, en $1992^{12}$, mais pleinement dans son temps en affirmant ainsi dès l'entame son appartenance au " camp républicain », elle rejoint ainsi une vaste et dominante catégorie, au moins sur un plan formel, de constitutions. Cette référence s'entend initialement par opposition à une forme monarchique ${ }^{13}$, entendue comme le gouvernement d'un seul tandis que dans la République, selon Aristote la souveraineté est entre les mains du peuple ${ }^{14}$. Dans ces conditions, l'accession à l'indépendance nationale vaut naturellement rétablissement du peuple dans ses droits et fondation républicaine. Bien qu'elle ne soit pas à l'origine synonyme de démocratie, la République tend au fil des siècles à s'y confondre dans la mesure où le peuple est à la source de tous pouvoirs, à telle

enseigne que de nos jours « il n'y a qu'une seule et unique maison : la République démocratique ${ }^{15}$ ». Tel est de prime abord le sens à donner à la double assertion d'un «État souverain et démocratique dénommé République » comme le fait l'article $1^{\mathrm{er}}$ de la Constitution mauricienne.

Près d'un quart de siècle de germination semble être un laps de temps suffisant pour que s'ancre un régime républicain chassant l'ombrelle de la monarchie couvrant l'indépendance tant le modèle de Westminster 16 fut prégnant. Ce modèle subi (I), donnant corps au mimétisme constitutionnel souvent à l'œuvre et observé dans les États nouvellement indé- pendants se vérifie ici. Le système constitutionnel mauricien ayant apporté la preuve de sa solidité et de sa stabilité s'est progressivement émancipé du giron juridique britannique en optant pour un régime républicain, preuve cette fois d'un modèle choisi (II) au risque d'accentuer le syncrétisme constitutionnel mauricien.

\section{I- L'INDÉPENDANCE DANS LA DÉPENDANCE : LE MODÈLE SUBI DU PARLEMENTARISME BRITANNIQUE}

Il est sans doute vain ici « d'émettre (après coup) des critiques faciles quoique fondées sur ce "néocolonialisme constitutionnel", il serait intellectuellement plus honnête d'admettre que ce "mimétisme constitutionnel" était à l'époque, et dans un premier mouvement d'émancipation, inévitable ${ }^{17} . .$. . Il s'agit seulement de constater la forte proximité entre la Constitution d'un État nouvellement souverain et l'ancienne métro- pole, d'autant plus qu'elle porte un modèle matriciel d'une grande catégorie du droit constitutionnel : le régime parlementaire (A). Toutefois, et c'est un trait dominant du système juridique mauricien, un syncrétisme constitutionnel agit en profondeur résultant d'une influence continentale (B).

\section{A - UN RÉGIME PARLEMENTAIRE À L'OMBRE DE WESTMINSTER}

«By way of introduction, the State and the Constitution have been defined in chapter one. Mauritius has been described as a sovereign democratic State and the Constitution as the supreme law of Mauritius ${ }^{18}$. » Il est vrai que le chapitre I, L'État et la Constitution, comporte deux articles inauguraux affirmant respectivement : «L'île Maurice est un État souverain et démocratique »; «La Constitution est la loi suprême ». Cette affirmation d'une souveraineté, non qualifiée de nationale ou populaire à rebours de la tradition constitutionnelle française est le siège de la reconnaissance de l'indépendance du nouvel État. À l'évidence la stratification sociale de l'île Maurice en est à l'origine. En outre, compte davantage le statut de l'État que la forme du régime, tant son essence souveraine a la priorité sur ses conditions d'existence. Comment ne pas y voir la manifestation imparfaite du tropisme indien ? Dans son Préambule, la Constitution de l'Union indienne du 26 janvier 1950 ne retient-elle pas que l'Inde est constituée d'une Sovereign Democratic Republic ? Ainsi le voisinage entre les caractères souverains et démocratiques d'un État accédant à l'indépendance pour évident qu'il soit ne se recommande pas d'une même évidence 
s'agissant de son caractère républicain. Pour le dire autrement, une révolution manque en l'occurrence à la fondation républicaine, les épisodes sanglants de janvier 1968 n'en relevant pas ; l'Indépendance fruit d'une évolution se déploie à l'ombre de la Monarchie quand la Révolution est souvent encline à enfanter la République ! Par ailleurs et formellement, la structure sociale de l'Inde dominée par une hiérarchie fondée sur l'appartenance communautaire n'est pas incompatible avec un régime républicain réduit à son sens le plus étroit et historique : la non- dévolution héréditaire du pouvoir. La parenté en la matière avec la société mauricienne laisse entrevoir un horizon républicain aux implications limitées s'agissant du statut individuel. Pour l'heure cet horizon est bouché par la plume du constituant, J. Colom soulignant que cet article $1^{\text {er }}$ a été « écrit par les juristes anglais selon le style des règlements coloniaux ${ }^{19}$ » comme le démontre l'absence de Préambule.

En attendant qu'une éventuelle réforme constitutionnelle ne se dessine, la Cour suprême a insufflé, certes tardivement, une vitalité toute juridique au caractère démocratique du jeune État. La Cour suprême a commencé par donner une substance à l'affirmation de la démocratie en la reliant d'une part au principe de l'État de droit et d'autre part à celui de la séparation des pouvoirs entre le législatif, l'exécutif et le judiciaire durant les années $1980^{20}$. Quelques années plus tard, le Conseil Privé considère à sa suite : « The idea of democracy involves a number of differents concepts. The first is the people must decide who should govern them. Secondly, there is the principle that fundamental rights should be protected by an impartial and independent judiciary. Thirdly, in order to achieve a reconciliation between the inevitable tensions between these ideas, a separation of powers between the legislature, the executive and the judiciary is necessary ${ }^{21}$. » Alors que pendant de longues années le terme « démocratique » ne possédait qu'une valeur purement déclaratoire, injusticiable, la jurisprudence a rendu possible cette observation : « The provision that Mauritius 'shall be... a democratic State'is an operative and binding provision ${ }^{22}$. » En somme, la lecture que fait le juge du terme « démocratie » recouvre parfaitement le sens étymologique et traditionnel d'un «gouvernement par le peuple».

Le substrat démocratique de la Constitution mauricienne se concilie sans mal avec la nature de son régime. Comme si sa chrysalide était insuffisamment achevée, le jeune État mauricien emprunte au colonisa- teur son système institutionnel, classiquement organisé en un régime parlementaire ${ }^{23}$. La Constitution de 1968 prévoit alors que le «pouvoir exécutif à l'île Maurice s'incarne dans Sa Majesté », laquelle est Chef de l'État, représentée par un gouverneur général, nommant à son tour le Premier ministre (article 59). En réalité, ce dernier, comme les membres de son cabinet, est issu du parti majoritaire, dont le leader aspire précisé- ment à diriger le cabinet. L'article 59-3 de la Constitution précise que le Gouverneur général nomme comme « Premier ministre le député qui lui paraît susceptible d'obtenir le soutien de la majorité des membres de l'Assemblée ». Dans ces conditions, le profil du régime se précise : "Our system of gouvernment is the system of cabinet government ${ }^{24}$.»

Du côté législatif, le Parlement est « composé de Sa Majesté et d'une assemblée législative ${ }^{25}$ ». Ce jardin à l'anglaise heurte le cartésianisme continental : dans un État nouvellement proclamé souverain le représentant d'une monarchie étrangère est à la fois une incarnation exécutive et détenteur d'une fonction législative. À ce titre, le gouverneur général détient une faculté d'empêcher, chère à Montesquieu, dans la mesure où les projets de loi votés par l'Assemblée législative doivent être approuvés par lui. Il reste que ce n'est point le gouverneur qui gouverne, mais le cabinet et que ce dernier tire sa force autant que sa légitimité de la majorité de l'Assemblée législative. À nouveau, les particularismes de la société mauricienne sont à l'origine d'un système électoral spécifique ${ }^{26}$. Sont en revanche communs les rapports entretenus entre le cabinet et l'Assemblée, il est « collectivement responsable » devant celle-ci, tandis que 
le « gouverneur général, agissant conformément à l'avis du Premier ministre, peut à tout moment proroger ou dissoudre 1'Assemblée " ${ }^{27}$. Comme l'analyse alors Louis Favoreu : " Le système britannique a été fidèlement transposé ${ }^{28}$. » Le constituant de 1968 pousse la fidélité jusqu'à expurger de toute référence républicaine le texte fondateur, laquelle aurait pu emporter des conséquences sur les structures de la société mauricienne.

Il serait vain à 50 ans de distance de souligner plus en avant la filiation de la Constitution mauricienne avec le parlementarisme britannique ; le propos vise davantage à en souligner le syncrétisme. À cet égard, il importe de déceler les éléments d'une influence continentale irriguant la norme suprême.

\section{B - UNE CONSTITUTION SOUS INFLUENCE CONTINENTALE}

Bien qu'il ne faille pas exagérer la distinction entre constitution écrite et coutumière pour ranger tout entière la Constitution britannique dans la seconde, il est certain que la Constitution mauricienne appartenant à la première catégorie, par ce seul élément penche du côté du modèle continental. Si le défaut d'un procédé de démocratie semi-directe prenant la forme d'un référendum - ne faisant toujours pas partie de la culture politique du pays ${ }^{29}$ - est patent, l'enjeu des élections générales de 1967 découle du lien entre leur résultat et la proclamation le cas échéant de l'indépendance assortie de la mise en œuvre d'une nouvelle constitution. Toutefois, et à nouveau, la prégnance coloniale est ici marquée dans la mesure où le texte constitutionnel n'est pas le fruit de la délégation du pouvoir constituant à l'assemblée élue, mais celui d'une écriture experte par Stanley Alexander de Smith, professeur britannique de droit public, qui a joué un rôle central au sein de la conférence constitutionnelle formée en particulier de représentants des partis poli- tiques mauriciens. En d'autres termes, la chaîne des événements illustre « le procédé d'élaboration non démocratique de cette écriture constitutionnelle soumise à une autorité coloniale ${ }^{30}$ ».

L'influence continentale se fait plus sensible ${ }^{31}$ dans le chapitre II de la Constitution consacré à la « Protection des droits fondamentaux et des libertés individuelles ». Tant sur le fond que sur la forme l'inspiration de la Convention européenne de sauvegarde des droits de l'homme ${ }^{32}$ et des libertés fondamentales de 1950 - et dans une moindre mesure de la Déclaration universelle des droits de l'homme 33 - est manifeste : protection du droit à la vie (art. 4), protection contre l'esclavage et le travail forcé (art. 6), garantie des droits de la défense (art. 10), liberté de conscience (art. 11), liberté d'expression (art. 12), liberté de réunion et d'association (art. 12) protection contre toutes formes de discrimination (art. 16). D'autres influences européennes se font sentir, ainsi la « liberté de fonder des établissements scolaires ${ }^{34}$ », relayées par l'article $14^{35}$, évoque inévitablement l'article 7 de la loi fondamentale retenant : « Le droit de fonder des écoles privées est garanti. » Il ne s'agit pas ici de tisser systématiquement les liens unissant la constitution mauricienne avec tel ou tel texte ou constitution européenne, en l'occurrence allemande, pour en souligner une parenté niée ou occultée, mais d'en éclairer la substance que l'on peut qualifier de républicaine, avant même que celle-ci ne fasse l'objet d'une reconnaissance formelle ou, pour céder aux travers langagiers du temps, d'en extraire l'ADN républicain.

Au demeurant, ce chapitre II est très fourni, formé des articles 3 à 19, plusieurs fois amendé, son écriture l'éloigne des standards habituels par le degré de précision de ses dispositions et par leur teneur. Certains passages abordent ainsi la question du « paiement des allocations de retraite à tout membre ou ancien membre de l'Assemblée nationale ${ }^{36} »$, ce qui au passage soutient l'analyse de Georges Vedel selon lequel : «Il n'existe pas de définition matérielle de la Constitution. Est constitutionnelle, quel qu'en soit l'objet, toute disposition émanant du pouvoir constituant ${ }^{37}$. » Quoi qu'il en soit, cet ensemble de droits et libertés 
bénéficie d'une protection juridictionnelle assurée par la Cour suprême ${ }^{38}$. Si le « système de justice constitutionnelle en vigueur dans la Constitution de la République de Maurice peut être essentiellement rattaché au modèle américain ${ }^{39}{ }$, cette juridiction assure un contrôle de constitutionnalité des lois suivant les contours d'un système présent de longue date dans les États du Commonwealth ${ }^{40}$. Plus largement et à ses débuts la Cour suprême participe d'une conception toute britannique du pouvoir judiciaire, comme en témoigne spécifiquement le maintien de l'institution du Comité Judiciaire du Conseil Privé ${ }^{41}$. Il s'agit depuis que la Constitution mauricienne a fait le choix de la République du plus visible des reliquats de sujétion juridique à l'endroit de la Couronne.

La Constitution mauricienne fait suivre la Protection des droits fondamentaux et des libertés individuelles d'un chapitre III «Citoyenneté », illustratif des spécificités historiques et géographiques de Maurice quant à son peuplement et son appartenance à la Couronne britannique ; la citoyenneté mauricienne emportant appartenance au statut de citoyen du Commonwealth. Le régime électoral mauricien, et au-delà la vie politique, « repose largement sur l'institutionnalisation du pluralisme culturel » et sur la prise en compte de communautés ethniques, ce qu'on désigne « sous le terme de communalisme » ${ }^{42}$. Ce pluralisme ethnique qui est la marque de la société mauricienne ne va pas sans heurts ${ }^{43}$. L'originalité des dispositions relatives à la citoyenneté mauricienne est également à la fois le témoin constitutionnel de l'accession à l'indépendance dans la mesure où elles opèrent une césure à la date de sa proclamation quant à l'acquisition de cette citoyenneté et le reflet du développement d'une population, l'éligibilité étant suspendue aux termes de l'article 33 à une relative maitrise de la langue anglaise qu'il faut être capable de parler et de lire «suffisamment bien » pour prétendre être membre de l'Assemblée législative ${ }^{44}$.

Les chapitres suivants sont respectivement consacrés au Gouverneur général (Chapitre IV), au Parlement (Chapitre V), à l'exécutif (Chapitre VI), à l'Assemblée régionale de Rodrigues (Chapitre VI A), au pouvoir judiciaire (Chapitre VII), aux Commissions judiciaire et légale et de la fonction publique ainsi qu'à cette dernière (Chapitre VIII), à l'Ombudsman (Chapitre IX), aux finances (Chapitre X) et enfin, assez classiquement le dernier chapitre (XI) traite de questions diverses et hétéro- gènes. En dépit ou grâce à son extraction teintée de colonialisme, la Constitution entrée en vigueur en 1968 n'a pas démenti le pronostic conclusif de Louis Favoreu : «L'île Maurice sera la démocratie parlementaire de l'océan Indien, si elle parvient à survivre ${ }^{45}$. » Mieux serait-on tenté d'ajouter, elle va devenir une République dans l'océan Indien.

\section{II- LE SYNCRÉTISME CONSTITUTIONNEL ASSUMÉ : LE MODÈLE CHOISI DU RÉGIME RÉPUBLICAIN}

Dans son avant-propos au recueil contenant la Constitution de la République de Maurice, objet d'une nouvelle traduction justifiée par une série de révisions au cours des années 1980 et au début des années 1990, Louis Favoreu mesure le chemin parcouru par les institutions politiques mauriciennes un quart de siècle plus tard et se félicitant de leur continuité, gage d'une réussite aux yeux du constitutionnaliste d'autant qu'elles ont su épouser les contours de leur temps au gré des révisions ${ }^{46}$, il ne manque pas de relever que la plus récente à ce moment-là « a changé même la nature de l'État en faisant passer Maurice du statut de monarchie à celui de République 47 ». Cette mutation constitutionnelle vaut mue institutionnelle, ou pour le dire autrement, un modèle chasse l'autre (A). Toutefois, pour éviter de céder à un nominalisme trompeur, masquant un républicanisme $f u z z y$, la République doit s'incarner pour acquérir sa propre substance, au besoin en mêlant les modèles (B). 


\section{A - L'AVÈNEMENT DE LA RÉPUBLIQUE MAURICIENNE : UN MODÈLE CHASSE L'AUTRE}

La première tentative avortée en décembre 1983 de révision constitutionnelle visant à inscrire le caractère républicain de l'État mauricien dans la Constitution s'inscrit dans le prolongement d'une aspiration émergeant au milieu des années 1970 dans les partis de gauche ou récemment créés comme le Mouvement Militant Mauricien (MMM). La période suivante est également marquée par des dissensions quant au régime politique adossé à la République. Faut-il qu'elle s'acclimate au modèle de Westminter ou bien doit-elle aller chercher l'inspiration dans un contre-modèle forgé à partir de l'exemple français ? Ces interrogations augmentées de divisions internes au camp républicain sont à l'origine du « parcours difficile 48 » conduisant à l'avènement de la République, comme l'atteste le retrait d'un amendement en ce sens en août 1990, quand « avant le vote [...] on s'est rendu compte qu'il manquerait probablement une voix pour qu'il soit adopté ${ }^{49} »$. En définitive, il faut une courte attente d'un an pour que soit promulgué l'Acte $n^{0}$ 48, Republic Constitutional amendments, ajoutant au caractère démocratique de l'État mauricien un caractère républicain.

Dans l'histoire politique française, la République est le costume institutionnel et juridique de la profonde aspiration du peuple français à l'égalité, sans qu'il soit besoin ici de renvoyer à sa devise. Or, pour paraphraser Jaurès, elle porte en elle l'égalité comme la nuée l'orage, si bien que cette dimension a largement contribué à la plus large adhésion possible quant à son adoption au début des années 1990. L'appel à la République a prospéré sur l'application limitée du principe d'égalité ${ }^{50}$. Ainsi, alors que dix ans plus tôt la revendication républicaine " apparais- sait comme révolutionnaire ou à tout le moins provocatrice. En 1992, elle fait l'objet d'un consensus général ${ }^{51} »$, après que les élections générales de 1991 aient permis l'émergence d'une majorité politique favorable à cette transformation ${ }^{52}$.

Cette transformation de l'État mauricien en République permet de tester un certain nombre d'idées reçues ou de tendances observées s'agissant de l'influence exercée par l'environnement juridique de l'ancien colonisateur sur l'État nouvellement indépendant. En premier lieu, «l'influence du droit diminue nécessairement et fatalement avec le temps ${ }^{53}$ ». L'assertion se vérifie ici, la monarchie constitutionnelle étant chassée par la République, avec ce contrepoint, « qu'on ne peut non plus négliger qu'au fur et à mesure que s'éloigne le souvenir de l'ancienne domination coloniale et des luttes nécessaires pour s'en délivrer, les vieilles rancunes s'évanouissent et, avec elles, le souci de rompre avec les codes légués ${ }^{54}$ ». D'évidence, la colonisation par le Royaume-Uni ayant succédé à celle exercée par la France, le recul de celui-là bénéficie à celle- ci. Le droit mauricien n'est pas ainsi exclusivement le fruit d'un alliage entre le droit britannique et français en matière privée ${ }^{55}$, mais également s'agissant du droit public.

D'autres idées reçues peuvent être confrontées à l'expérience mauricienne ${ }^{56}$, et parmi celles-ci l'une mérite que l'on s'y attarde, dans le prolongement de la précédente : elle " consiste à imaginer que l'influence du droit français dans les pays anciennement colonisés, tiendrait en quelque sorte à l'impulsion reçue à l'époque de la colonisation et serait donc nécessairement condamnée à s'atténuer avec le temps $57 »$. Bien entendu, il convient de se garder de tout anachronisme, le constituant mauricien ne puise pas dans la République agonisante sous les coups de butoir du Consulat et dévoyée par l'Empire, les sources de son inspiration au temps où l'île était française (de 1715 à 1814). De sorte qu'à proprement parler, l'ancien colonisé ne reprend pas, à la différence de 1968, le modèle du colonisateur du temps de son emprise, il s'agit davantage de s'inscrire dans une tradition constitutionnelle à laquelle est assimilé un lointain colonisateur. 
Il reste, qu'à l'instar de nombreux États africains, on assiste « au retour des forces des mécanismes caractéristiques de l'ancienne métro- pole, marqués par la domination d'un président élu au suffrage universel, par un Premier ministre qui joue le plus souvent de rôle de fusible, par deux assemblées fortement contrôlées grâce aux procédures caractéristiques du parlementarisme rationalisé ${ }^{58} \ldots$ ». Certes, la Constitution mauricienne est loin de reprendre fidèlement ces divers traits distinctifs du système politique et constitutionnel français, il n'en demeure pas moins qu'elle participe à sa manière à ce surgeon juridique français dans l'océan Indien. Faut-il pour autant en relativiser la portée à la manière de Charles Cadoux jugeant que « le passage de la monarchie constitutionnelle (britannique) à la République Mauricienne [...] ne change rien au fonctionnement du régime ${ }^{59}{ }$ ? En réalité, si le constituant a pu céder au nominalisme, les vertus performatives du droit constitutionnel ne sont plus à démontrer en sorte que l'adjonction du caractère républicain au caractère démocratique de l'État est susceptible de donner corps à une République démocratique.

\section{B - DONNER CORPS À LA RÉPUBLIQUE : DE MODÈLE EN MODÈLE}

Au regard des particularités de la Constitution originale, tenant en particulier dans l'existence d'un Gouverneur général, l'affirmation d'une République mauricienne s'accompagne de changements constitutionnels y afférents. C'est ainsi que cette figure de l'ancien monde, est remplacée presque à la symétrie de notre temps par le président de la République, de la manière la plus simple qu'il soit, le chapitre IV de la Constitution consacré au Gouverneur général abrite à partir du 12 mars 1992 les dispositions relatives au président de la République. D'une certaine manière, l'exécutif devient autocéphale en ce sens qu'il ne s'ancre pas sur la Couronne par l'intermédiaire du Gouverneur général. L'article 58-1 pose ainsi : «Le Président est investi du pouvoir exécutif de Maurice. » Cette rupture (cette coupure du cordon ombilical monarchique serait-on tenté d'écrire) est, avec l'affirmation fondamentale de l'article $1^{\text {er }}$, la manifestation la plus sensible de la transformation du régime ; l'Exécutif n'a plus son siège dans l'ancienne métropole ${ }^{60}$, mais dans la fonction présidentielle. Sur un registre identique, l'article 28 prévoit qu'il « est le Chef de l'État et le Commandant en Chef de la République de Maurice».

Un corps politique et institutionnel est donné au régime républicain mauricien, encore faut-il que cette incarnation nouvelle ait une certaine épaisseur. Suivant les principes classiques des régimes parlementaires, le Président tire sa légitimité de l'Assemblée qui l'élit à la majorité de ses membres sur proposition du Premier ministre. La durée de son mandat, renouvelable sans limitation, épouse la législature puisqu'elle est de 5 ans. De sorte que le processus de légitimation offre le profil d'une pyramide inversée, le Président procède de l'Assemblée - tout comme le Premier ministre et le gouvernement laquelle procède à son tour des citoyens ${ }^{61}$. De manière originale, un Vice-Président, désigné de la même manière que le Président «exerce les fonctions que le Président lui attribue ${ }^{62}$ ». Sa présence comme l'expression plus haut de « Com- mandant en Chef » dit assez l'influence du régime présidentiel étatsunien. Ce régime parlementaire est coiffé d'un exécutif emblématique du régime présidentiel ; la créature du constituant est faite d'une tête présidentielle greffée sur un corps parlementaire. Le parallèle s'impose d'autant plus qu'une procédure de révocation à l'emprise élargie peut être engagée aussi bien à l'encontre du Président que du Vice Président sans préjudice d'un régime d'immunité protecteur ${ }^{63} ;$ l'immunité n'est pas comme chacun sait l'impunité.

Toutefois, la présence originale de l'attelage Président/Vice-Président ne doit pas tromper, la réalité du pouvoir exécutif relève du Premier ministre, chef de la majorité parlementaire, nommé par le Président toujours en sa qualité de « député qui lui semble le plus susceptible d'obtenir le soutien de la majorité des 
membres de l'Assemblée ${ }^{64} »$. S'agissant de la formation du gouvernement, le pouvoir de nomination revient formellement au Président « sur avis conforme du Premier ministre ${ }^{65}$ ». Ce parlementarisme classiquement moniste reste le trait dominant de ce syncrétisme constitutionnel ou plus que la figure d'un président de la République « à la française » s'impose à la tête de l'État celle d'un équivalent monarchique, le Président préside, mais ne gouverne pas. L'observation ressort du devoir d'information qui pèse sur le Premier ministre pour « tout ce qui a trait à la conduite générale du Gouvernement de Maurice ${ }^{66}$ ». Au fond sa plus importante prérogative tient, comme le monarque constitutionnel, dans une dissolution de l'Assemblée qu'il peut à tout moment prononcer « sur avis conforme du Premier ministre ».

Au final, il ressort d'une lecture comparée entre la Constitution de 1968 et celle de 1992 que le constituant a procédé à un changement (que l'on qualifierait en langage sportif de poste pour poste) entre le Gouverneur général et le président de la République. Au point que l'article consacré à la suppléance du premier (art. 29 de la Constitution) ouvre la voie à la fonction de Vice-Président.

La faible densité constitutionnelle de la fonction présidentielle nourrit des propositions de réforme. Loin d'être l'alpha et l'oméga de la vie politique et institutionnelle, à tout le moins peut-il prétendre incarner un point fixe dans le ciel constitutionnel mauricien. Pour ce faire, comme l'analyse Milan J. N. Meetarbhan : «One major area of reforme that should be considered is the mode of election of the President. » Une réflexion sur sa désignation s'est ainsi engagée, " the mode of election of the President must be reviewed if an allocation of power leading to a healthier democracry is to be sustained ${ }^{67} \gg$. Alors qu'en France les propositions de révision visant à rééquilibrer les pouvoirs au sein de l'exécutif au bénéfice du Premier ministre, en raison de sa responsabilité politique et de son défaut s'agissant du chef de l'État, se fanent aussi rapidement qu'elles fleurissent depuis près de 30 ans, à Maurice une perspective inverse prévaut. L'objectif vise ici à assurer un transfert de pouvoir au sein du pôle exécutif favorable au président de la République, consécutif à un accroisse- ment de sa légitimité. En d'autres termes, la réforme du mode de désignation du Chef de l'État est un moyen, non une fin. Difficile à envisager en présence du représentant de la Couronne, elle tend à faire de la fonction présidentielle un pôle de stabilité, dominant de sa hauteur le gouvernement et l'Assemblée. De sorte que cet alizé de réforme soufflant sur Maurice renvoie à la $\mathrm{V}^{\mathrm{e}}$ République des débuts, celle du Président arbitre au-dessus des partis et en tout état de cause détaché de la majorité parlementaire pour mieux échapper à son emprise.

Toutefois, il est à craindre qu'à l'instar de la République française, l'élection du président de la République au suffrage universel direct ne vienne substituer un déséquilibre à l'autre. Cette éventualité n'a pas dissuadé le Parti travailliste, alors dans l'opposition, de proposer en décembre 1991 un tel procédé. À nouveau et à la faveur des élections générales de 2014, « an alliance of two of the main political parties campaigned on a proposal to amend the Constitution to provide for election of the President by universel suffrage and confer additional powers of the President ${ }^{68} \gg$. Pour autant, une élection au suffrage universel direct est susceptible d'attiser des fractures interethniques. Aussi, les constitutionnalistes mauriciens voient-ils dans la Constitution de Singapour prévoyant l'élection du Président dans un environnement culturel et ethnique comparable un modèle opérationnel susceptible d'acclimatation ${ }^{69}$. Il reste que la voie est étroite entre l'actuel mode de nomination du président de la République et l'onction populaire. Un suffrage indirect forgé sur un corps électoral formé de grands électeurs dépassant le cadre législatif est une option médiane et comme telle raisonnable sous réserve précisément de la timidité sur laquelle elle prospère. Quoi qu'il en soit, il s'agit bien dans un cas comme dans l'autre d'éviter une concentration du pouvoir exécutif. Point ici l'ancienne et persistance méfiance des mœurs républicaines à l'endroit d'un exécutif trop puissant. 
À l'heure où l'île Maurice célèbre le cinquantième anniversaire de sa Constitution, les regards se tournent vers son aîné de dix ans, la Constitution du 4 octobre 1958 fondant la Ve République. Au terme de ce mouvement, la première renvoie à la seconde le reflet de sa jeunesse idéalisée et doit être avertie de ses dérives. La force d'un modèle tient à sa plasticité et pour avoir su habilement combiner les influences, la Constitution mauricienne a atteint une durée que peuvent lui envier de nombreux États du sud-ouest de l'océan Indien et de l'Afrique australe ; quand le modelé devient à son tour modèle. À cette condition, toutefois, que le Château du Réduit ne perde de son harmonie architecturale à trop vouloir mêler le Palais de l'Élysée à celui de Westminster.

\section{Notes de bas de page}

1. L. Favoreu, L'île Maurice, Encyclopédie politique et constitutionnelle, Institut international d'administration publique, Paris, Berger Levrault, 1970, p. 7.

2. Issu de l'amendement ${ }^{\circ}$ IV, Act $\mathrm{n}^{\circ}$ 48, 1991 : Government Gazette of Mauritius, Legal Supplement, 1991-12-23, no 131, p. $231 s q$.

3. Pour la période antérieure à l'indépendance v. Les Constitutions de l'île Maurice (1723-1961), textes réunis par D. Napal, Mauritius archives publications, Port-Louis, Mauritius Printing Compagny, 1962.

4. V. L. Favoreu, L'île Maurice, op. cit., p. 25-29.

5. Adopté par le Parlement britannique le 29 février 1968.

6. En ce sens, J. Colom, «L'écriture dirigée par le Colonial officiel de la Constitution mauricienne de 1968 », in J. Colom (dir.), Le développement constitutionnel dans les États du sud-ouest de l'océan Indien, Aix-en-Provence, PUAM, 2013, p. 24 sq.

7. M. J. N. Meetarbhan, «L'évolution de la Constitution mauricienne depuis 1968 », Annuaire des Pays de l'océan Indien, vol. XIV, 1995-1996, p. 25.

8. J.-L. Quermonne, « République », in O. Duhamel et Y. Mény (dir.), Dictionnaire consti- tutionnel, Paris, Puf, 1992, p. 921.

9. Sur le processus politique et constitutionnel conduisant à l'indépendance : M. Akung,

«Vie politique et évolution constitutionnelle de Maurice - 1958-1968 », in Le développement constitutionnel dans les États du sud-ouest de l'océan Indien, op. cit., p. 43 sq.

10.Pour la démocratie : P. Bouretz, in Dictionnaire constitutionnel, op. cit., p. 283 sq. ; pour la République : J.-L. Quermonne, op. cit., p. 921 sq.

11.L'article $1^{\mathrm{er}}$ de la constitution iranienne double le caractère républicain d'une référence religieuse : «The form of government of Iran is that of an Islamic republic ». La conception européenne de la République $\mathrm{y}$ voit un oxymore dans la mesure où elle s'est construite en opposition avec des régimes monarchiques appuyés par des religions d'État. Une observation comparable vaut pour de nombreuses républiques dites populaires passées ou présentes, dont celle de Chine (art. ${ }^{\text {er }}$ de la Constitution).

12.Les travaux de la doctrine consacrés à la Constitution mauricienne et plus encore au passage de la monarchie à le République sont limités aussi bien en langue anglaise que française. Pour la fondation de la Constitution mauricienne il convient de se reporter à l'ouvrage précité de L. Favoreu paru en 1970. On peut se référer à l'ensemble des travaux de J. Colom, inauguré par sa thèse soutenue en 1989 sous la 
direction de L. Favoreu : La justice constitutionnelle dans les États du nouveau Commonwealth : le cas de l'île Maurice, Aix- en-Provence, PUAM, Paris, Economica, 1994. Pour la constitution républicaine : M. J. N. Meetarbhan, « L'évolution de la Constitution mauricienne depuis 1968 », Annuaire des Pays de l'océan Indien, vol. XIV, 1995-1996, p. 23-40 et son ouvrage paru à l'occasion du cinquantenaire : Mauritian Constitutional Law. Constitution of Mauritius with Commentaries, Port-Louis, Precigraph Limited, 2017.

13.« À sa naissance la république était l'absence d'un roi. » M.-P. Deswarte, Essai sur la nature juridique de la République, Paris, L'Harmattan, 2003, p. 71.

14.Aristote, La politique, livre III, chapitre V.

15.G. Zagrebelsky, « Cinquante ans d'activités de la Cour constitutionnelle italienne »,

$R D P, 2007, \mathrm{n}^{\circ} 1$, p. 138.

16.V. B. de Smith, «Exporting the Westminster Model and the Constitution of Mauri- tius », in Mutations constitutionnelles dans l'océan Indien, Colloque international, université de

La Réunion, Faculté de droit et des sciences économiques et politiques, 6, 7 et 8 avril, 1994 ; Annuaire des pays de l'océan Indien, vol. XIV, 1995-1996, p. 41 sq.

17.C. Cadoux, « Panorama des situations constitutionnelles dans la zone océan Indien »,

Annuaire des pays de l'océan Indien, vol XIII, 1993-1994, p. 263.

18.S. Bhuckory, Our constitution, Port-Louis, The Mauritius Printing Company, 1971, p. 10.

19.J. Colom, « L'écriture dirigée par le Colonial Office de la Constitution mauricienne de 1964 », op. cit., p. 37.

20.V. B. Domah, La Cour suprême et la construction de la démocratie à l'île Maurice, in Le développement constitutionnel dans les États du sud-ouest de l'océan Indien, op. cit., p. 83 sq.

21.The State v. Khoyratty (Mauritius) Rev 1 [2006] UKPC 13 (22 March 2006), pt. 12.

22.M. J.N. Meetarbhan, « The Constitution at 50 », in R. Ramtohul and T. H. Eriksen, The Mauritian Paradox: Fifty years of Development, Diversity and Democracy, Réduit, University of Mauritius Press, 2018, p. 17.

23.L. Favoreu, op. cit., p. 36.

24.S. Bhuckory, op. cit., p. 37.

25.Article 31-1 de la Constitution.

26.V. P. G. Rajendra, « Droit électoral. Projet de réforme électorale prospective en droit constitutionnel ou la pratique du Best Loser System et du système proportionnel », RFDC, 2009, nº 2, p. $431 s q$.

27.Article 51-1 de la Constitution

28. Op. cit., p. 40.

29. J. Chan Low, «L'actualité du débat constitutionnel dans la vie politique à l'île Maurice », in $L e$ développement constitutionnel dans les États du sud-ouest de l'océan Indien, op. cit., p. 72.

30. J. Colom, « L'écriture dirigée par le Colonial Office de la Constitution mauricienne de 1964 », op. cit., p. 24.

31. «In so far as basic rights and freedoms are concerned, the present Constitution draws inspiration from both English law and the European Convention for the Protection of Human Rights and Fundamental Freedoms. » D. Ramsewak, The Constitution, its legal aspect and political philosophy, Port-Louis, Proag printing limited, 1991, p. 15. Sur cette question de l'écriture du chapitre II, v. J. Colom, « L'écriture dirigée par le Colonial Office de la Constitution mauricienne de 1964 », op. cit., p. 28 sq.

32. V. V. Boolell, « The Influence of the European Convention on the Constitutional Law of Mauritius », European Human Rights Law Review, 1996, n 1, p. 159 sq.

33. V. tableau réalisé par R. P. Gunputh et L. Sermet, « ̂̂le Maurice et Seychelles », $R F D C, 2013, \mathrm{n}^{\circ} 4$, p. 1028. 
34. Article 3 b) de la Constitution.

35. « De la liberté de fonder des écoles ».

36. Article 171 a) de la Constitution.

37. « Schengen et Maastricht constitutionnelle », RFDA, 1992, $\mathrm{n}^{\circ}$ 2, p. 178. Au renfort de sa démonstration, le doyen Vedel cite la mention dans la Constitution suisse de dispositions relatives aux abattoirs ainsi que sous la III ${ }^{\mathrm{e}}$ République, la loi constitutionnelle du 10 août 1926, complétant la loi constitutionnelle du 25 février 1875 traitant de l'autonomie de la caisse de gestion des bons de la défense nationale et d'amortissement de la dette publique.

38. V. J. Colom, La justice constitutionnelle dans les États du nouveau Commonwealth : le cas de l'île Maurice, op. cit., p. 162 sq.

39. X. Philippe, « Les mutations constitutionnelles dans l'océan Indien. Les modèles de justice constitutionnelle », Annuaire des Pays de l'océan Indien, vol. XIII, 1992-1994, p. 304.

40. V. J. Colom, op. cit., p. 24-28.

41. L'article 81 de la Constitution traite du pourvoi devant le Comité judiciaire (Judicial Committee). V. R. P. Gunputh, «Le Comité judiciaire du Conseil Privé : atouts, enjeux et conséquences », in Le développement constitutionnel dans les États du sud-ouest de l'océan Indien, op. cit., p. 101 sq.

42. C. Boudet, «L'émergence de la démocratie consociative à Maurice », in Annuaire des Pays de l'océan Indien, XVII (2001-2002), p. 325.

43. V. C. Boudet, «Émeutes et élections à Maurice. La mort de Kaya, aléa ou échec de la construction nationale ?», Politique africaine, 2000, $\mathrm{n}^{\mathrm{0}} 3$, p. $153 \mathrm{sq}$.

44. Cette disposition est demeurée inchangée depuis 1968.

45. Op. cit., p. 49.

46. Pour celles intervenues entre 1968 et 1993, v. J. Colom, op. cit., p. 206 sq.

47. La Constitution de la République de Maurice en versions anglaise et française, Port- Louis, Best Graphic Ltd, 1993, p. vi.

48. J. Colom, op. cit., p. 217.

49. M. J. N. Meetarbhan, «L’évolution de la Constitution mauricienne depuis 1968 », op. cit., p. 30.

50. V. J. Colom, op. cit., p. 165 sq.

51. C. Cadoux, op. cit., 283.

52. V. J. Chan Low, «Vie politique et élections générales de 1948 à 1965 », in

J.-M. Jauze (dir.), L'île Maurice face à ses nouveaux défis, Paris, L'Harmattan, 2008, p. 71-72.

53. A. et D. Cabanis, «L'influence du droit français liée au processus de colonisation- décolonisation », RJOI, $2005, \mathrm{n}^{\circ} 5$, p. 11.

54. Idem.

55. V. S. Rohlfing-Dijoux, «Droits français et anglais réconciliés dans une synthèse originale du droit des affaires mauricien », in numéro spécial, Le rayonnement du droit français dans le monde, RJOI, 2005, $\mathrm{n}^{\circ}$ 5, p. 65 sq.

56. Comme celles touchant au caractère linéaire des évolutions ou au faible rôle joué par des influences personnelles, A. et D. Cabanis, op. cit., p. 11.

57. Idem, p. 25.

58. Idem, p. 23-24.

59. Op. cit., p. 283. X. Philippe estime pareillement : la «physionomie de l'île Maurice ne s'en est pas trouvée bouleversée », « Mutations et révisions constitutionnelles dans les pays de l'océan Indien », Annuaire international de justice constitutionnelle, 1994, vol. X, p. 158. 
60. L'article 58-1 dans sa version de 1968 retenait : « Le pouvoir exécutif à l'île Maurice s'incarne dans sa Majesté ».

61. Cette formulation emprunte au célèbre discours de Bayeux du 16 juin 1946 : «C'est donc du chef de l'État, placé au-dessus des partis, élu par un collège qui englobe le Parle- ment mais beaucoup plus large et composé de manière à faire de lui le Président de l'Union française en même temps que celui de la République, que doit procéder le pouvoir exécutif ».

62. Article 27-2 b) de la Constitution.

63. Article 30 et 30 A de la Constitution. V. M. J. N. Meetarbhan, Mauritian Constitutio-nal Law..., op. cit., p. $153-157$.

64. Article 59-3 de la Constitution.

65. Idem.

66. Article 65 de la Constitution.

67. M. J. N. Meetarbhan, « The Constitution at 50 », op. cit., p. 26.

68. V., M. J. N. Meetarbhan, Mauritian Constitutional Law..., op. cit., p. 150. 69. Idem, p. 151-152. 